\title{
Evolutionary constraints in fitness landscapes
}

\author{
Luca Ferretti $^{1}$ - Daniel Weinreich ${ }^{2}$. Fumio Tajima ${ }^{3}$. Guillaume Achaz ${ }^{4,5}$
}

Received: 29 January 2018 / Revised: 1 June 2018 / Accepted: 3 June 2018 / Published online: 11 July 2018

(c) The Genetics Society 2018

\begin{abstract}
In the last years, several genotypic fitness landscapes—combinations of a small number of mutations-have been experimentally resolved. To learn about the general properties of "real" fitness landscapes, it is key to characterize these experimental landscapes via simple measures of their structure, related to evolutionary features. Some of the most relevant measures are based on the selectively acessible paths and their properties. In this paper, we present some measures of evolutionary constraints based on (i) the similarity between accessible paths and (ii) the abundance and characteristics of "chains" of obligatory mutations, that are paths going through genotypes with a single fitter neighbor. These measures have a clear evolutionary interpretation. Furthermore, we show that chains are only weakly correlated to classical measures of epistasis. In fact, some of these measures of constraint are non-monotonic in the amount of epistatic interactions, but have instead a maximum for intermediate values. Finally, we show how these measures shed light on evolutionary constraints and predictability in experimentally resolved landscapes.
\end{abstract}

\section{Introduction}

Fitness landscapes have been a very successful metaphor to study evolution. Most simply, the idea of Sewall Wright (1932) to view evolution as a hill-climbing process proved to be appealing and inspired a vast amount of theoretical work in phenotypic and molecular evolution (Orr 2005; de Visser and Krug 2014).

In evolutionary biology, fitness landscapes have been used to study adaptation. In the classical metaphor, an evolving population is abstracted into a particle that navigates in the landscape (Orr 2005). In a strong selection weak mutation regime (Gillespie 1983), populations are restricted

Luca Ferretti

luca.ferretti@gmail.com

$\triangle$ Guillaume Achaz

guillaume.achaz@mnhn.fr

1 The Pirbright Institute, Woking, UK

2 Ecology and Evolutionary Biology, Brown University, Providence, USA

3 University of Tokyo, Tokyo, Japan

4 Institut de Systématique Evolution, Biodiversité, ISYEB, UMR 7205 CNRS MNHN UPMC EPHE, Paris, France

5 Centre Interdisciplinaire de Recherche en Biologie, CIRB, UMR 7241 CNRS Collège de France INSERM, Paris, France to follow evolutionary paths of increasing fitness, which can sometimes even be completely deterministic.

The smoothness or ruggedness of the fitness landscape affect many fundamental features of adaptation and evolution in the landscape, and especially the repeatability of the adaptive process (e.g. Kauffman 1993; Colegrave and Buckling 2005; Chevin et al. 2010; Salverda et al. 2011). Consequently, it is now clear that several aspects of evolutionary processes directly depend on the structure of the fitness landscapes on which the organisms are evolving. Furthermore, Wright's idea of genotype-fitness landscapes moved from a metaphor to an object of experimental studies, as several fitness landscapes were experimentally resolved (Malcolm et al. 1990; Whitlock and Bourguet 2000; Lunzer et al. 2005; O'maille et al. 2008; Bridgham et al. 2009; Lozovsky et al. 2009; de Visser et al. 2009; Hall et al. 2010; da Silva et al. 2010; Tan et al. 2011; Chou et al. 2011; Khan et al. 2011; Flynn et al. 2013; Jiang et al. 2013; Meini et al. 2015; Mira et al. 2015; Palmer et al. 2015; Bank et al. 2016), including the seminal work of Weinreich et al. (2006) on antibiotic resistant mutations (see de Visser and Krug 2014, for a review). In that regard, characterizing the structure of experimental and model fitness landscapes is a key step in our ability to decipher evolution at the finest scale.

Experimental fitness landscapes make it possible to test predicted properties of evolutionary trajectories through evolution experiments, together with sequencing (Achaz 

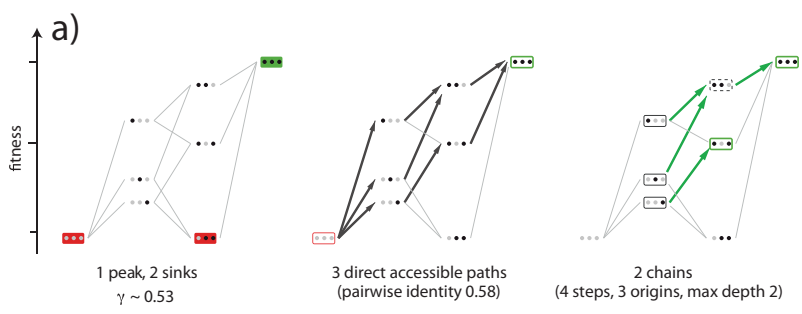

Fig. 1 Measures for fitness landscapes. We depict, on an illustrative fitness landscape with three loci of two alleles, the three sets of measures used in this study. a Peaks, here in green, are genotypes with no fitter neighbors whereas sinks, here in red, are genotypes with only fitter neighbors. In this landscape, $\gamma$ (the correlation between fitness effects of mutations in neighboring genotypes) is 0.53 . b there are three direct accessible paths ending at the global peak and starting at its antipode. All paths pass through four genotypes and share the start and endpoint; moreover, two paths share another genotype as well, hence the average fraction of shared genotypes is $(2 / 4+2 / 4+3 / 4) / 3=0.58$. c The two chain trees are composed of four steps (genotypes with a single fitter neighbor), three origins (two for one chain, one for the other) and have a maximal depth (the largest number of steps chained together) of two

et al. 2014). Currently available fitness landscapes are based on mutations in a few loci, typically 4-10 (Szendro et al. 2013; Weinreich et al. 2013). Since the number of genotypes scales as the product of the number of alleles at all loci, testing all the combinations of mutations in an organism (or even in a protein) is beyond the reach of any reasonable future experiment. However, small landscapes have been resolved and analyzed. An exciting opportunity raised by the recent release of these experimental landscapes is to find the adequate model(s) that is (are) able to generate synthetic model landscapes that share similar features with the observed ones. In that regard, characterizing the structure of small fitness landscapes is a key step for our understanding of evolution in the presence of biological interactions among mutations. These epistatic interactions determine the shape of fitness landscapes.

Epistasis is one of the most relevant features of fitness landscapes. By definition, the (Malthusian) fitness effect of a combination of multiple non-interacting mutations corresponds to the sum of fitness effects of the individual mutations. Hence, epistasis can be defined as any departure from additivity of the fitness effects. Additive landscapes, i.e. landscapes where the fitness effects of all mutations are additive, are the only non-epistatic landscapes. In these landscapes evolution is unconstrained, i.e. populations are always able to reach the highest fitness peak through a sequence of beneficial mutations; exchanging the order of these mutations simply results in another fitness-increasing path to the peak. Other landscapes show a variable amount and structure of epistatic interactions, which may act as evolutionary constraints.

As for other genomic data, the high dimensionality of fitness landscapes make them almost impossible to visualize (although some attempts were proposed, see McCandlish 2011; Brouillet et al. 2015). As a consequence, the analyses of fitness landscapes will mostly rely on measures that capture important features of their structures. Several measures were proposed previously and used to analyze experimental fitness landscapes (reviewed in Szendro et al. 2013; Stadler 2002). The most natural one that was historically used to appreciate the ruggedness of a landscape is its number of peaks (Weinberger 1991). Intriguingly, although ruggedness is more adequately represented by both types of extrema, only little attention has been payed to the number of sinks (genotypes with only fitter neighbors). We therefore suggest that peaks and sinks are both adequate measures of the landscape ruggedness (Fig. 1a). Most models generate synthetic landscapes with the same mean number of peaks and sinks; however, small landscapes can have a different number of peaks and sinks due to random sampling. Other measures such as $r / s$ ratio (ratio of the roughness over additive fitness, see Szendro et al. 2013), fraction of sign epistasis and correlation of fitness effects (Ferretti et al. 2016), as well as path-based measures, such as the number of accessible paths to the highest peak (Weinreich et al. 2006), were also proposed to characterize the structure of fitness landscapes. As all these represent direct or indirect measures of epistasis, all these measures were shown to be pairwise correlated in experimental (Szendro et al. 2013) and model fitness landscapes (Ferretti et al. 2016). In that regard, other measures related to evolution but somehow uncorrelated to the amount of epistasis would be welcome to investigate the nature and the structure of the interactions in the landscapes.

In this study, we propose two new sets of measures, related to the short-term predictability of evolutionary outcomes.

The first set looks at the relation of accessible paths. In fact, while the number of accessible paths is recognized as a very important measure of epistasis and constraints (Weinreich et al. 2006), it contains no information on the similarity between these paths (Lobkovsky et al. 2011). The distance between paths is relevant for short-term predictability. In fact, evolution is more predictable when different paths tend to visit the same genotypes. For this reason, one natural measure of path similarity is the average fraction of genotypes visited by both paths (Fig. 1b).

The second set of measures is related to successions (chains) of obligatory beneficial mutations, that are paths going through genotypes with a single fitter neighbor. Their aim is to quantify the amount of evolutionary constraints in the landscapes. In the limit of strong selection and low mutation rates, when a hill climbing evolutionary path reaches a genotype that has a single fitter neighbor, the next step of the path is essentially deterministic. In this case, only one of the mutations available in the landscape can improve 
the fitness. If several genotypes with a single way out are connected together they form a chain of "obligatory" mutational events that often ends on a peak (Fig. 1c), but could also end on an intermediate genotype.

Chains form a tree structure of obligatory fitnessincreasing paths, already noticed in Lobkovsky et al. (2011). We describe several measures on this tree that can be used to characterize the structure of fitness landscapes (Fig. 1c). These measures have been chosen for two reasons. First, they have an immediate interpretation in biology in terms of evolutionary constraints. Second, for small landscapes, these chain measures are only weakly correlated with epistasis, hence they can bring independent information on the structure of the interactions.

In the following, we will define these evolutionary measures in detail, then present analytical and numerical results for experimental and model fitness landscapes and finally discuss their relations with the existing measures of epistasis.

\section{Definitions}

\section{Similarity between accessible paths}

Many fitness landscape measures of epistasis are correlated across experimental (Szendro et al. 2013) and model (Ferretti et al. 2016) fitness landscapes with the amount and strength of epistasis. However, one could envision that landscapes with similar measures of epistasis could still be quite different in terms of their structure of epistatic interactions, as well as their evolutionary properties. New measures should be sensitive to these differences in landscape structure and the corresponding differences in evolution, while being possibly uncorrelated with changes in fitness effects across different backgrounds or other direct measures of epistasis. Measures that are directly related to properties of evolutionary trajectories are the most promising candidates in this respect.

Here, we focus on a class of statistics that measure the amount of constraints on the possible evolutionary paths. These constraints can take different forms. A well-known example is the reduction in the number of selectively accessible (i.e. fitness-increasing) paths to the highest peak from its antipode in the landscape. The number of accessible paths and the number of accessible direct paths (without any reversion) are classical measures of epistatic constraints, since all the direct paths are accessible in additive landscapes, and their number decreases with epistasis (Weinreich et al. 2006). Interestingly, the probability of finding a direct path has been analytically studied in some models (Franke and Krug 2012; Schmiegelt and Krug
2014; Hegarty et al. 2014; Berestycki et al. 2016; Hwang et al. 2017).

However, the number of accessible paths may not tell the full story. Indeed, one could imagine that epistatic constraints decimate paths at random or, on the contrary, only paths traversing subregions of the genotype space. In the latter case, the constraints would not only reduce the number of accessible paths, but also concentrate the remaining paths across specific genotypes, hence increasing predictability of the evolution.

For biallelic landscapes, we propose a simple measure of these constraints, namely the average pairwise fraction of shared genotypes between two direct accessible paths to the highest peak from its antipode (Fig. 1b). This is an intuitive measure of constraints in the landscape as two accessible paths that often pass through the same genotypes will tend to explore the same regions of the genotype space. The larger the identity among selectively accessible trajectories, the better our ability to predict the steps of an adaptive walk. This measure is reminiscent of previous approaches based on path divergence (Lobkovsky et al. 2011) and on path distances/entropies (Manhart and Morozov 2014), but it is more focused on local constraints rather than on global constraints.

This measure can be generalized to multiallelic landscapes by taking the average of the biallelic measure over all biallelic sublandscapes containing the highest peak. Note that the biallelic measure is undefined when there is either no path or a single path to the highest peak.

\section{Chains of obligatory steps}

Another natural measure of evolutionary constraints is given by the abundance and structure of maximally constrained paths in the whole landscape. To characterize these paths in a simple and effective way, we focus on genotypes with only a single beneficial mutation.

All fitness-increasing paths that pass through such genotype will share this mutation. Some landscape have "chains" of consecutive mutations with this property (see Fig. 1c). We now examine the abundance and size of these chains as measures of evolutionary constraints.

We define a chain step as a mutation $g \rightarrow g^{\prime}$ that is the only possible fitness-increasing mutation from the genotype g. Chain steps can occur one after another, forming a linear path of obligatory mutational steps, that we call a chain. Several chain steps can lead to the same genotype, but a genotype can have at most one outgoing chain step. For this reason, chains can form tree-like structures, that we call a chain tree. A chain tree is formally the set of all genotypes, which are forced to evolve along obligatory paths up to a common final genotype, i.e. a maximal set of connected chain steps. This definition implicitly assumes a strong 

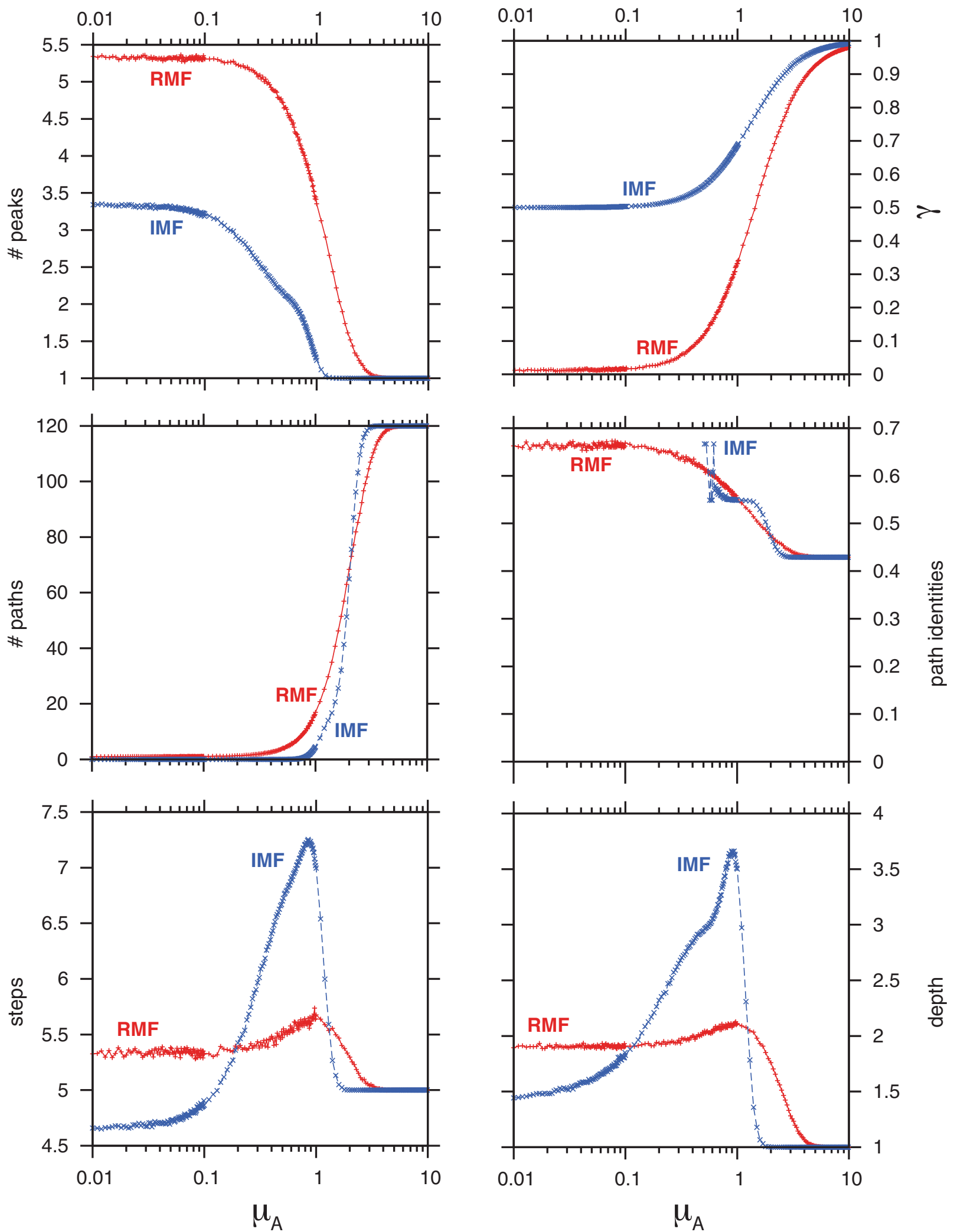

Fig. 2 Epistasis and chain measures for different landscape models with $L=5$. The landscapes are build from $10^{4}$ simulations of an additive component with mean $\mu_{\mathrm{a}}$ and variance $\sigma_{\mathrm{a}}=\mu_{\mathrm{a}} / 10$ and an epistatic component. The epistatic component is: (RMF) an HoC model with $\sigma_{\mathrm{HoC}}^{2}=1$; (IMF) an Ising model with mean incompatibility

cost $\mu_{\mathrm{c}}=1$ and variance $\sigma_{\mathrm{c}}^{2}=0.1$. We plot the amount of epistasis as measured by $\gamma$ and peaks, as well as the number and similarity of direct accessible paths plus the number of chain steps and their maximum depth 
a) RMF with 5 loci

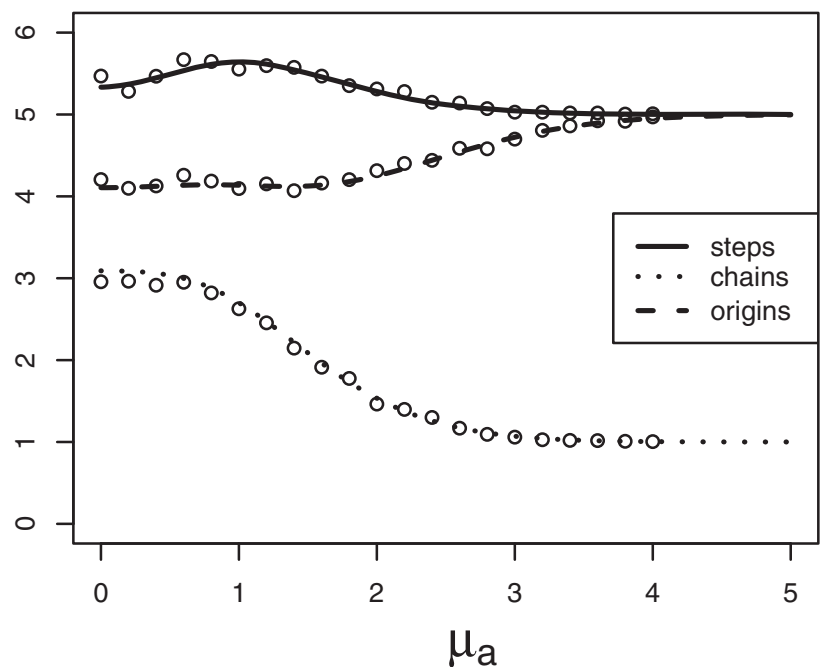

b) RMF with 8 loci

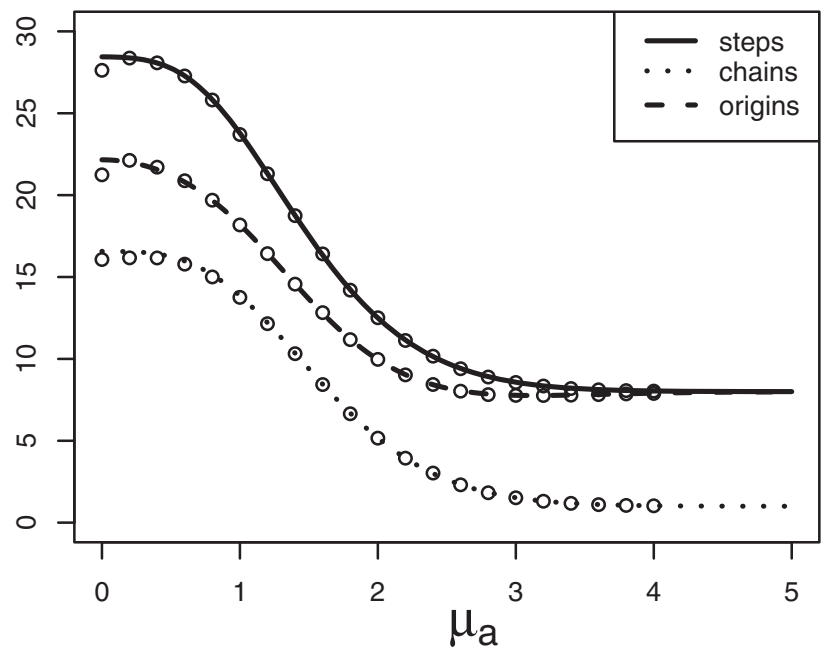

Fig. 3 Chain statistics versus epistasis in Rough Mount Fuji landscapes. Expected values for chain measures (number of steps, number of chains, number of origins) as a function of the additive fitness effect

selection regime (Gillespie 1983), as there is no fixation for deleterious or neutral mutations. In an additive landscape, there is a single chain tree containing only those genotypes one mutation away from the (single) peak.

Note that other landscapes can contain more than one chain tree (or none). The set of all chain trees correspond to the "tree component" of the landscape, i.e. the component with deterministic evolution, discussed sporadically in the literature (Lobkovsky et al. 2011; Kauffman and Weinberger 1989). We compute also the number of origins, that correspond to all genotypes that are initial points of a chain (obviously excluding intermediate steps). Finally, we compute also the maximal depth of all chains in the landscape,
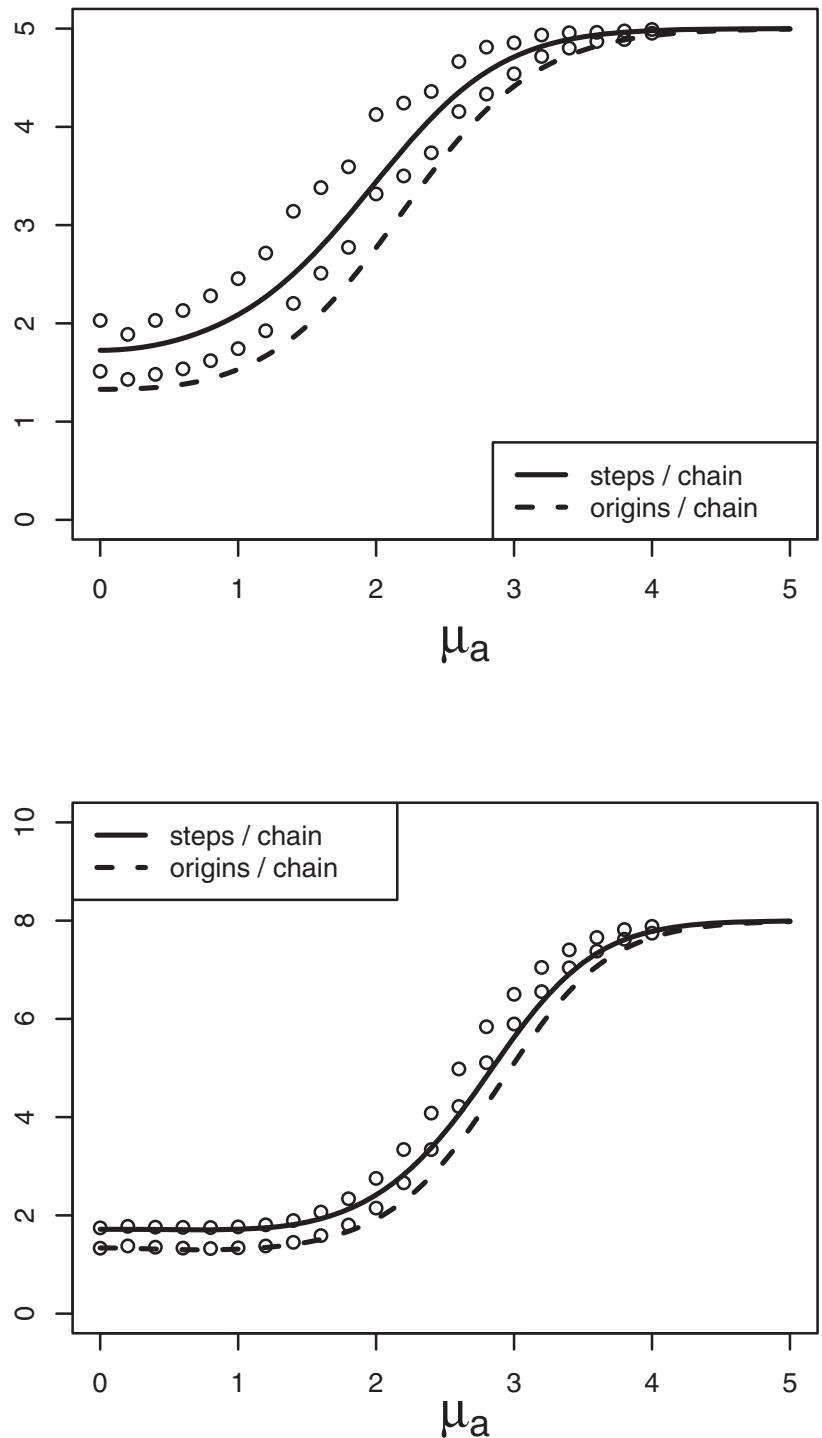

$\mu_{\mathrm{a}}$ for a RMF landscape with $\sigma_{\mathrm{a}}^{2}=0, \sigma_{\mathrm{HoC}}^{2}=1$. Lines represent the analytical mean values, while dots represent the average over $10^{4}$ simulations

that is, the maximum number of consecutive steps. In an additive landscape, the depth of the only chain tree is 1 .

\section{Model landscapes}

Extreme models of epistatic interactions are additive landscapes, corresponding to the case of no epistasis, and the HoC model (Kingman 1978), which corresponds to the case of strong epistasis. The latter model assigns random uncorrelated values to the fitnesses of the genotypes; these values are extracted from a given distribution, e.g. a Gaussian with variance $\sigma_{\mathrm{HoC}}^{2}$ and mean 0 . 
We compute the measures on accessible paths and tree chains on two different biallelic model landscapes:

- The Rough Mount Fuji (RMF) model is a mixture of an additive component and a random contribution from a HoC model. The additive component has fitness effects randomly chosen from a Gaussian distribution of mean $\mu_{\mathrm{a}}$ and variance $\sigma_{\mathrm{a}}^{2}$, while the HoC component is Gaussian with variance $\sigma_{\mathrm{HoC}}^{2}$. This RMF model is often used to interpolate between a complete additive landscape and a complete random one (Aita et al. 2000). We consider a fixed strength of the random "House of Cards" component; hence, the higher the mean additive effect of mutations $\mu_{\mathrm{a}}$, the more additive is the model; consequently, when $\mu_{\mathrm{a}} \rightarrow \infty$, the model becomes additive, whereas it converges to an House of Cards model when $\mu_{\mathrm{a}} \rightarrow 0$.

- The Ising Mount Fuji (IMF) model (Ferretti et al. 2016) was also introduced to mix an additive component (also with mean $\mu_{\mathrm{a}}$ and variance $\sigma_{\mathrm{a}}^{2}$ ) with a fixed Ising-like set of interactions in which each pairs of interacting loci implies a fitness cost if the two loci in the pair do not have the same allele. This cost is assumed to be Gaussian distributed with mean $\mu_{\mathrm{c}}$ and variance $\sigma_{\mathrm{c}}^{2}$ and it could be interpreted biologically as a genetic incompatibility between interacting alleles. In this model, all loci are located along a sequence and each locus interacts only with its neighboring loci.

We compute the mean value for the number of peaks, the number of direct paths and their identity, as well as chain steps and depth for RMF and IMF models, varying the additive contribution of the two models. Results are reported in Fig. 2. We computed also the $\gamma$ measure of epistasis (Ferretti et al. 2016), which is defined as the correlation of fitness effects of mutations before and after a single change in the genetic background. $\gamma=1$ corresponds to no epistasis while $\gamma=0$ corresponds to strong epistasis.

\section{Accessible paths}

In both models, the number of direct accessible paths is close to zero for strong random interactions $\left(\mu_{\mathrm{a}} \rightarrow 0\right.$ : HoC model), but it increases with increasing additive component $\mu_{\mathrm{a}}$ (i.e. decreasing epistasis) as expected, with a steep slope where the fitness variance due to the additive component is comparable to the epistatic one (see Fig. 2).

The similarity of accessible paths increases with the weight of random interactions (i.e. with epistasis) in both models. Since the number of direct paths follows the opposite trend, this suggests an inverse relation between number and similarity of paths. However, this relation is not necessarily informative on the structure of the epistatic interactions, rather it appears to be a common feature of small landscapes.

\section{Chains}

In an additive landscape, all the $L$ genotypes around the peak are origins of a chain tree that ends at the peak. This starlike chain tree of depth 1 around the peak is the only chain tree in these landscapes. In contrast, in a HoC model, there are many small and slightly deeper chain trees; their number is of the order of $2^{L} /(L+1)$ (see proof in the Appendix). Therefore, it would be tempting to conclude that the number of chain trees (as well as other measures of chains) are correlated to the amount of epistasis. However, this is not the case.

For example, in an RMF model with equal additive contributions, it is possible to derive exact and approximate theoretical results for the mean of some chain measuresmost relevant, the number of chain trees, chain steps and origins. Equations are presented in Appendix.

The results are illustrated in Fig. 3, where the mean of several chains measures are reported as a fraction of additive component in the RMF model. Furthermore, even though chain measures for the IMF model cannot be computed analytically, they can be retrieved from simulations (Fig. 2). Interestingly, several of the chain measures appear to be non-monotonic with respect to epistasis in the RMF model and even more so in the IMF model. This nonmonotonicity and the weak correlations suggest that these statistics depend weakly on epistasis.

Both the number of steps and the chain depth tend to have a maximum for intermediate values of epistasis, when the contributions of the additive component and of the interactions are comparable (Figs. 2 and 3). Interestingly the type of interaction plays a important role in the structure of the chains, as only short chains are observed for the RMF model, whereas long chains are observed for the IMF model. It is important to notice that the size of the landscape changes the dependence between epistasis and chain measures (depth and abundance) (Fig. 3).

\section{Experimental landscapes}

We computed the accessible direct paths (number and similarity) and the chain measure (number of chains, steps and origins, as well as maximum depth) on 38 experimentally biallelic resolved landscapes (Malcolm et al. 1990; Whitlock and Bourguet 2000; Lunzer et al. 2005; Weinreich et al. 2006; O'maille et al. 2008; Bridgham et al. 2009; Lozovsky et al. 2009; de Visser et al. 2009; Hall et al. 2010; da Silva et al. 2010; Tan et al. 2011; Chou et al. 2011; Khan et al. 2011; Flynn et al. 2013; Jiang et al. 2013; Meini et al. 


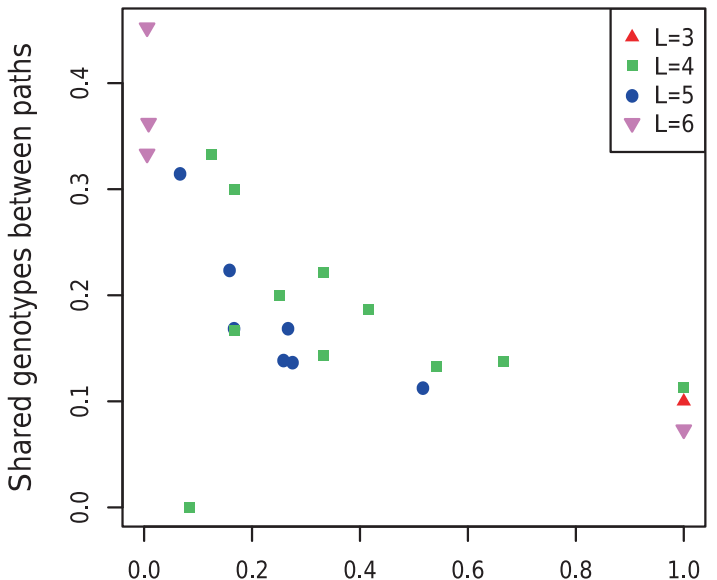

Fraction of accessible direct paths
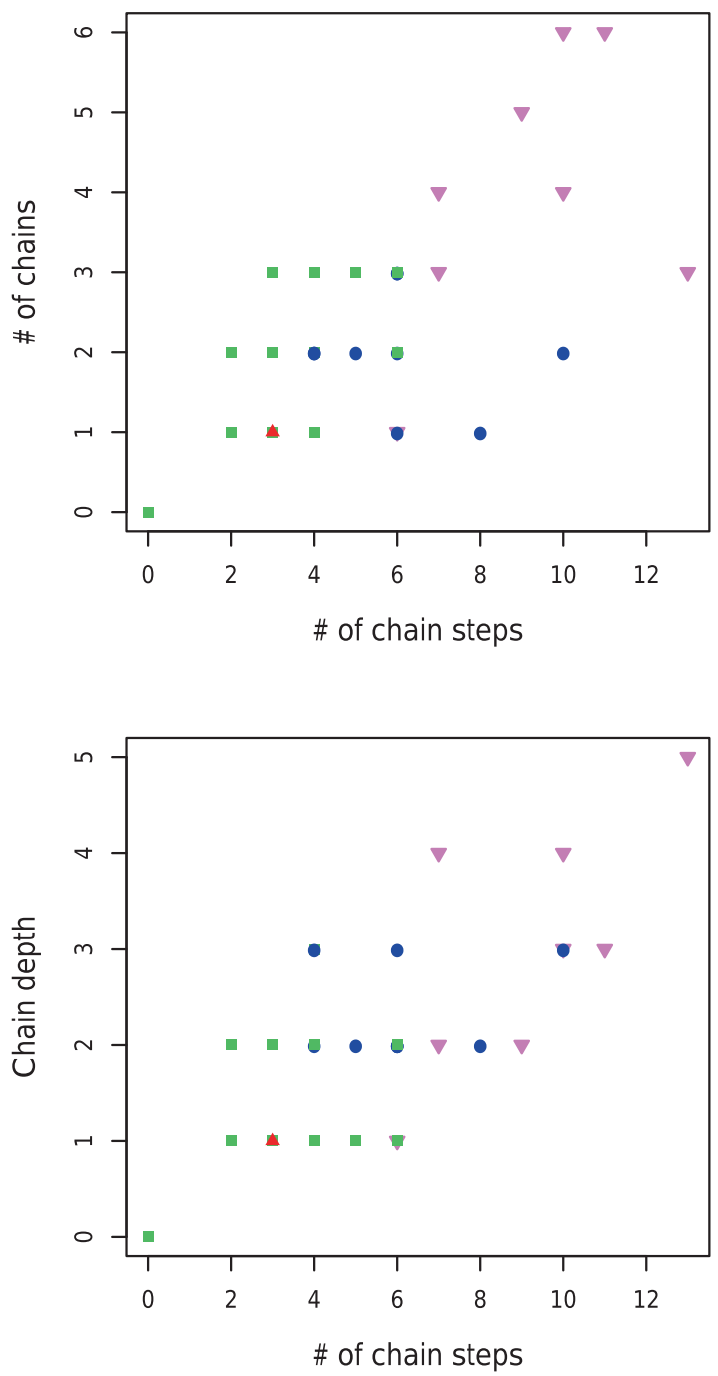

Fig. 4 Relation between landscape measures in several experimental biallelic landscapes with different number of loci. Top: Similarity between accessible direct paths (computed as path similarity minus its minimum possible value for the landscape) as a function of the fraction
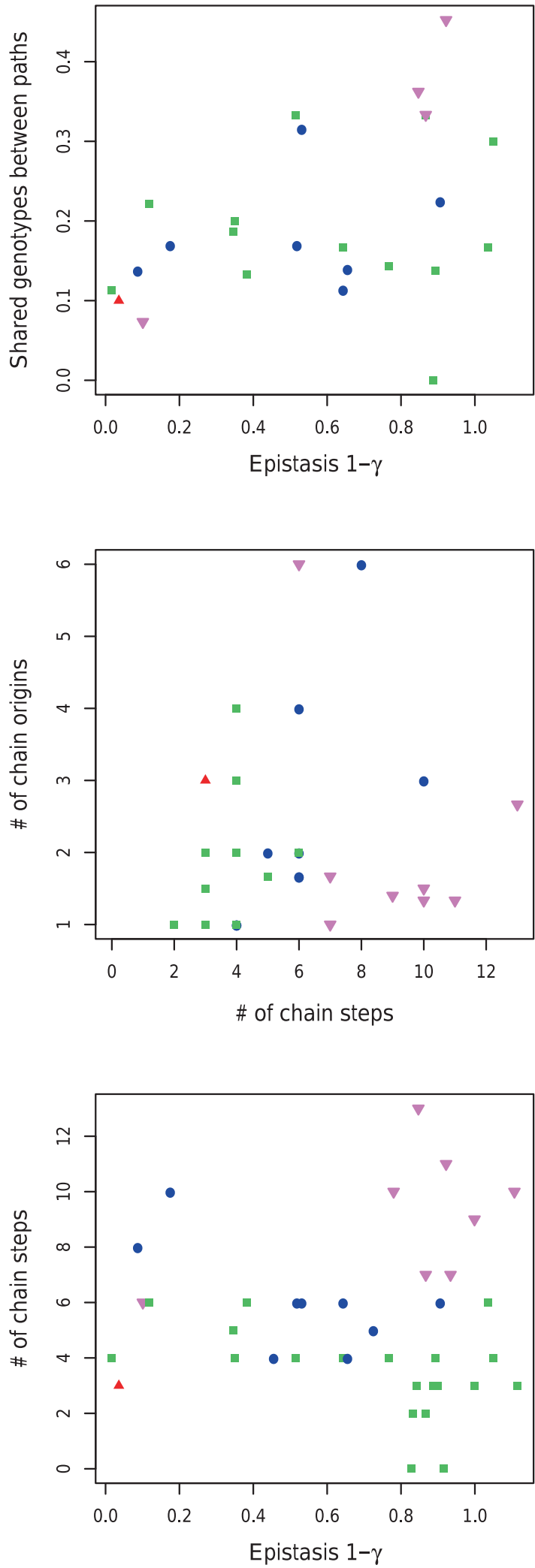

accessible direct paths and of epistasis (defined as $1-\gamma$ ). Middle and bottom: Number of chain trees, origins, and maximum chain depth versus the number of chain steps or $1-\gamma$ 
Weinreich et al., 2006

a)

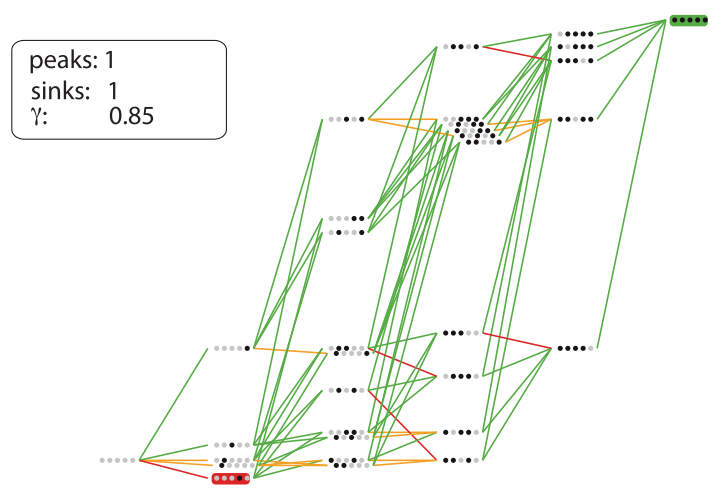

de Visser et al., 1997

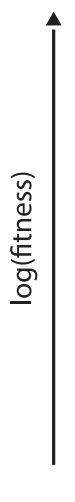

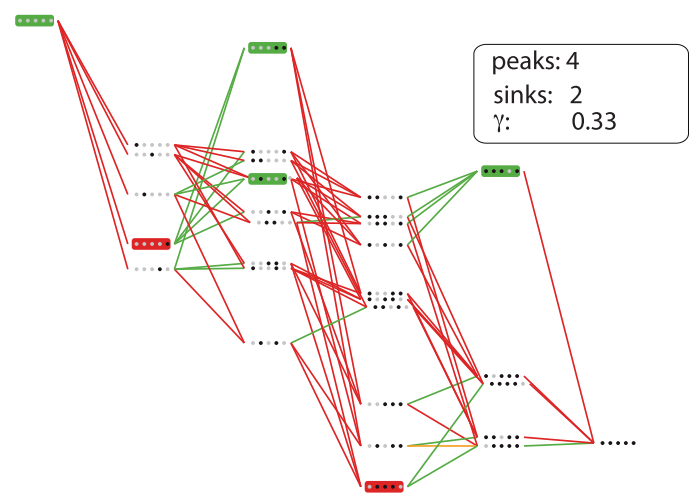

b)

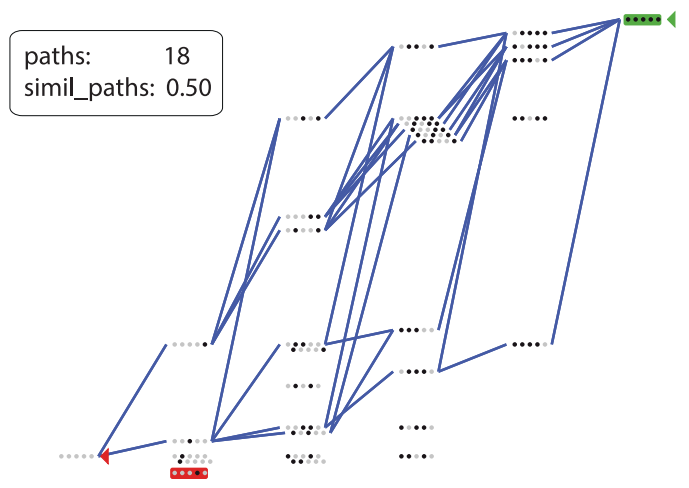

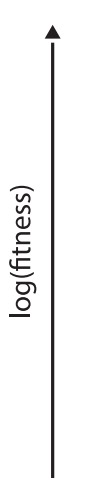

mis
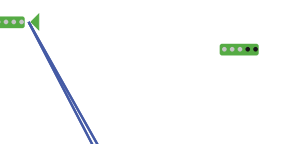

paths: 25

simil_paths: 0.54
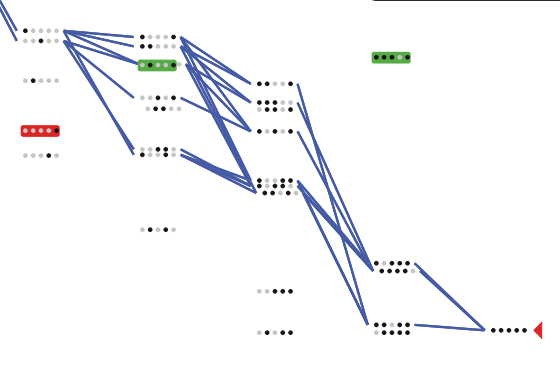

c)

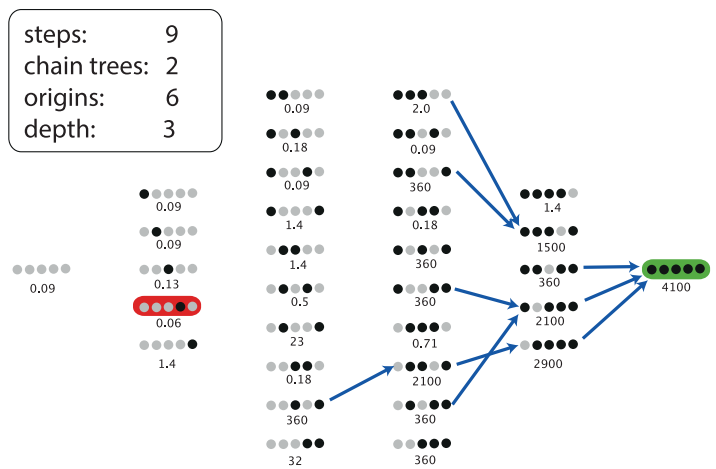

Fig. 5 Epistasis measures and their corresponding illustrations for two experimental fitness landscapes. The pictures and the statistics were computed using MAGELLAN (Brouillet et al. 2015). The left column represents different views from the Weinreich et al. (2006) landscapes, whereas the right one concerns the csI from de Visser et al. (1997). a On the top panel, we report the general aspect of the landscapes, as

2015; Mira et al. 2015; Palmer et al. 2015). They differ on their number of loci: 1 landscape has 3 loci, 20 have 4 loci, 9 have 5 loci, and 8 have 6 loci and their general features are summarized in Weinreich et al. (2017). Results can be seen in Fig. 4.

We also illustrate explicitly these statistics for two landscapes. The first landscape is the landscape of antibiotic (cefotaxim) resistance of $\beta$-lactamase mutations in an Escherichia coli plasmid from Weinreich et al. (2006) (Fig. 5 , left). The five mutations have a very strong effect that

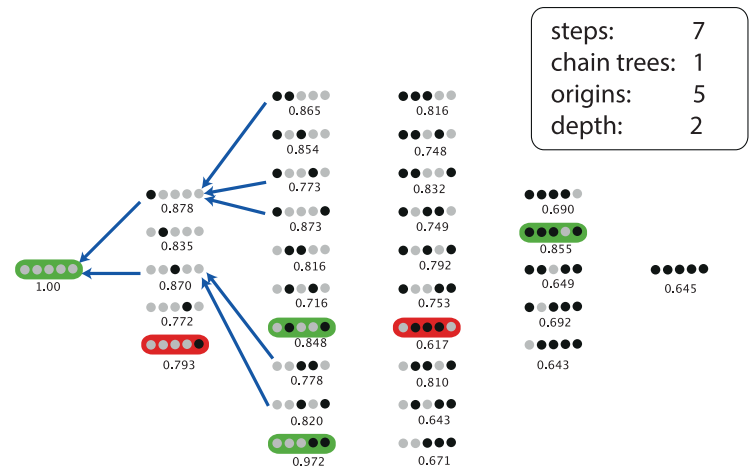

well as previous measures of epistasis. b On the middle panel, we depict and report the number of direct accessible paths and their average pairwise similarity. c Finally, we report four measures concerning chains of obligatory beneficial mutations, on a sky view of the landscapes

together give a $4 \times 10^{4}$ increase in antibiotic resistance and were therefore selected together. Given the huge selective advantage of the combined mutations, this landscape is single-peaked, where the peak corresponds to the five-point mutant. Furthermore, it also has a single sink, that interestingly does not correspond to the wild type, and is weakly epistatic $(\gamma=0.85)$.

The second is a landscape of deleterious mutations in Aspergillus niger from Franke et al. (2011) (Fig. 5, right). It is one of the four $L=5$ complete sublandscape (csI) of a 
Table 1 Correlation coefficients between landscape measures

\begin{tabular}{|c|c|c|c|c|c|c|c|}
\hline & peaks & sinks & $\gamma$ & paths & simil_path $^{\mathrm{a}}$ & steps & depth \\
\hline \multicolumn{8}{|c|}{ Experimental landscapes } \\
\hline peaks & 1 & - & - & - & - & - & - \\
\hline sinks & 0.45 & 1 & - & - & - & - & - \\
\hline$\gamma$ & 0.55 & 0.37 & 1 & - & - & - & - \\
\hline paths & 0.38 & 0.17 & 0.36 & 1 & - & - & - \\
\hline simil_path $^{\mathrm{a}}$ & 0.10 & 0.07 & 0.09 & 0.47 & 1 & - & - \\
\hline steps & $<0.01$ & 0.15 & $<0.01$ & 0.12 & $<0.01$ & 1 & - \\
\hline depth & 0.05 & 0.14 & $<0.01$ & $<0.01$ & $<0.01$ & 0.33 & 1 \\
\hline \multicolumn{8}{|l|}{ RMF model } \\
\hline & peaks & sinks & $\gamma$ & paths & simil_path & steps & depth \\
\hline peaks & 1 & - & - & - & - & - & - \\
\hline sinks & 0.57 & 1 & - & - & - & - & - \\
\hline$\gamma$ & 0.76 & 0.74 & 1 & - & - & - & - \\
\hline paths & 0.67 & 0.59 & 0.68 & 1 & - & - & - \\
\hline simil_path $^{\mathrm{a}}$ & 0.63 & 0.63 & 0.71 & 0.85 & 1 & - & - \\
\hline steps & 0.09 & 0.03 & $<0.01$ & $<0.01$ & 0.02 & 1 & - \\
\hline depth & 0.02 & 0.13 & 0.11 & 0.10 & $<0.01$ & 0.28 & 1 \\
\hline \multicolumn{8}{|l|}{ IMF model } \\
\hline & peaks & sinks & $\gamma$ & paths & simil_path & steps & depth \\
\hline peaks & 1 & - & - & - & - & - & - \\
\hline sinks & 0.67 & 1 & - & - & - & - & - \\
\hline$\gamma$ & 0.71 & 0.42 & 1 & - & - & - & - \\
\hline paths & 0.72 & 0.72 & 0.72 & 1 & - & - & - \\
\hline simil_path $^{\mathrm{a}}$ & $\mathrm{n} / \mathrm{a}$ & 0.70 & 0.59 & 1 & 1 & - & - \\
\hline steps & 0.19 & $<0.01$ & 0.05 & $<0.01$ & $<0.01$ & 1 & - \\
\hline depth & $<0.01$ & 0.11 & 0.04 & 0.24 & 0.02 & 0.70 & 1 \\
\hline
\end{tabular}

Spearman $\rho^{2}$ correlation of pairs of measures across 38 experimental landscapes (top panel) or $10^{4}$ random realizations of the RMF (medium panel) and IMF models (low panel) with $L=5, \sigma_{\mathrm{HoC}}=1$ (for RMF), $\mu_{\mathrm{I}}=1$ and $\sigma_{\mathrm{I}}=0.2$ (for IMF), $\sigma_{\mathrm{a}}=\mu_{\mathrm{a}} / 10$ and $\mu_{\mathrm{a}} \log$-uniformly distributed in $[0.01,10]$

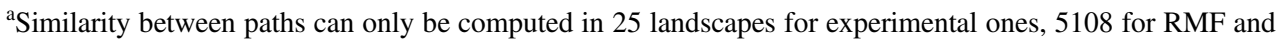
3242 for IMF, as the other ones have 1 or less accessible paths larger landscape with $L=8$ loci (de Visser et al.1997). This landscape is a combination of unrelated deleterious mutations where epistatic interactions were not filtered by natural selection. This landscape has 4 peaks and 2 sinks; in fact, at present it is one of the most rugged among the completely resolved landscapes $(\gamma=0.33)$.

\section{Accessible paths}

The results in Fig. 4 show a clear negative trend between the number of the paths and their similarity, which is observed across different numbers of loci and both across direct and indirect paths (not shown). This trend is predicted by landscape models and suggests that in small landscapes, epistatic interactions do not block evolutionary trajectories at random; instead, they constrain whole groups of similar trajectories in a clustered way, hence reducing the accessibility of the landscape but someway increasing the predictability of evolutionary trajectories.
The landscape by Weinreich et al. (2006) has 18 accessible direct paths to the highest peak (and a further 9 indirect accessible paths), out of 120 accessible paths for an additive landscape of the same size. Direct paths share slightly more than half of their genotypes ( 0.504 or 0.533 considering indirect paths as well), versus a minimum of 0.333 (i.e., only the extremes). Similarly, the landscape by de Visser et al. (1997) has 25 accessible paths (all are direct ones) that share on average 0.542 of their genotypes. Both landscapes appear to follow the same pattern outlined in Fig. 4 for the other empirical landscapes.

\section{Chains}

The number of chain steps is unrelated to measures of local epistasis, such as $\gamma$ (Fig. 4), supporting the arguments for a weak dependence of chain statistics on epistasis. Interestingly enough, however, this measure of the total size of chains in the landscape is related to several other chain 
measures, like the total number of chains, origins and the maximum chain depth, as shown in Fig. 4 (see also Table 1). For a fixed size of the landscape, these measures tend to increase slightly with the number of chain steps, meaning that landscapes with larger chain trees tend to have slightly more chains, and deeper and wider chains as well.

The landscape by de Visser et al. (1997) has a single chain of depth 2 with 5 origins and 7 steps, i.e. a slightly larger chain than expected according to the RMF model and to other empirical landscapes. This is not so surprising considering the amount of epistasis in this landscape, which could increase. On the other hand, the landscape by Weinreich et al. (2006) has a relatively small overall amount of epistasis, but it contains two chains with a maximum depth of 3,6 origins, and 9 steps, revealing one of the largest chain structures observed in empirical landscapes.

\section{Correlations between measures}

In this section, we discuss the relations existing between the newly proposed measures and the existing ones.

From a theoretical perspective, the number of chain steps is a close relative of other measures, such as the number of peaks and sinks, since they correspond to three different components of the distribution of beneficial mutations. This stems from the theory of fitness graphs. Fitness graphs correspond to a network of genotypes, where each pair of genotypes related by a beneficial mutation is connected by a directed link (Weinreich et al. 2005; Crona 2014). The outdegree of a node is defined as the number of outgoing links, i.e. the number of beneficial mutations from the corresponding genotype. The out-degree distribution (or equivalently, the distribution of the number of beneficial mutations from a genotype) is simply the distribution of the out-degrees of all nodes. Since there are no fitnessincreasing mutations from a peak, peaks are simply nodes with out-degree 0 . Therefore, the number of peaks is actually the first bin of the out-degree distribution. Similarly, in a biallelic landscape, the number of sinks correspond to the number of genotypes with out-degree $L$ (the last bin of the out-degree distribution). Chain steps correspond simply to nodes of out-degree 1 . Hence, the number of chain steps is the second bin of the out-degree distribution of the fitness graph, and is therefore a natural step further in the characterization of the out-degree distribution and the properties of fitness graphs.

However, this does not mean that number of peaks and of chain steps are necessarily correlated. In fact, we suggest that there is no direct relation between the amount of epistasis and the number of chains.
To evaluate in a more systematic way the relations between these and other measures, we perform a correlation analysis for the 38 experimental landscapes, as well as for $10^{4}$ random replicates of RMF and IMF model landscapes each. We select the number of peaks, the number of sinks, $\gamma$, as well as four of the measures discussed in this article: the similarity of direct accessible paths, plus the number of chain steps and the maximum chain depth. We compute the Spearman correlation coefficients of all pairs of measures in the experimental as well as in the RMF and the IMF landscapes varying the model parameters (in particular, the ruggedness).

The pairwise correlations (Table 1) confirm the intuition that most measures are all strongly correlated, including the similarity between paths. However, the correlation between most measures is notably low with both chain measures. This shows that the chain measures quantify some landscape properties that are not simply correlated to the amount of epistasis.

Interestingly, the lack of correlation of chains with other measures of epistasis is apparent when we compare them across different models of epistatic interactions. In Fig. 2, we depict how the different epistasis and chain measures depend on the amount of epistasis and the underlying epistatic model (HoC vs. Ising). The comparison between measures of epistasis and chains shows clearly that there is no simple relation between them, as these models of interactions have different behavior. The size of chains in the landscape depends strongly on the nature of the epistatic interactions and shows a non-monotonic behavior with the amount and strength of epistasis, as larger chains are more probable for intermediate levels of epistasis. Hence chain measures could provide useful information beyond the existing measures of epistasis.

\section{Discussion}

In this work, we presented two new sets of landscape measures, which have a simple interpretation and cover a range of potential applications. Both these measures are based on the sign of the fitness effects, i.e. on the fitness graph, hence they are well suited for landscapes where only fitness ranks or the beneficial/deleterious nature of the fitness effects can be experimentally determined. These measures and the others discussed here have been implemented in MAGELLAN, a graphical tool to explore small fitness landscapes (Brouillet et al. 2015).

The first set of measures - the fraction of genotypes shared between paths-is an interesting and revealing measure, but has several limitations. The first is precisely the strong anti-correlation between the number of accessible paths and their similarity, which reduces the usefulness of 
the latter as an independent measure, at least for small landscapes. The second is the impossibility to define path similarity in any meaningful way in cases where epistasis is so strong that there are no accessible paths, or just one. Finally, this measure is restricted to biallelic landscapes or sublandscapes.

The second set of measures is a more fine-grained measure of evolutionary constraints, based on genotypes with a single beneficial mutation available. These genotypes represent steps in "obligate" fitness-increasing paths on the landscape, funnelling different evolutionary paths through the same mutation. Sequences of such beneficial mutations represent chains of obligatory fitness-increasing mutations.

Chains are a natural choice for a measure of evolutionary constraints in the framework of fitness graphs. In particular, chain steps are strongly related to the distribution of beneficial mutations from a genotype, that is the out-degree distribution of the fitness graph corresponding to the landscape. In fact, as discussed before, there is a one-to-one correspondence between chain steps and genotypes with a single fitness-increasing mutation. The number of fitnessincreasing mutations from a given genotype is the outdegree of the genotype in the fitness graph. Therefore, chain steps are simply nodes of out-degree 1 . In this respect, this statistics is a close relative of one of the most well-studied measures of fitness landscapes, namely the number of peaks, which correspond to genotypes without beneficial mutations (i.e. nodes of out-degree 0 ).

Both the number of steps and related measures like the depth of chains have an immediate evolutionary interpretation, in terms of evolvability and constraints, yet they show peculiar properties. The most relevant one is that they are often non-monotonic or weakly correlated with the amount of epistasis (i.e. measured by the number of peaks, sinks or $\gamma$ ), as we have shown using simulations and analytically. The lack of correlation between epistasis and chains shows that these new statistics can be used to obtain independent information about the nature of the interaction, instead of their abundance.

Interestingly, the number of chain steps, the chain depth (Fig. 2) and even the total number of accessible paths (Szendro et al. 2013) seem to be peaked at intermediate weight of epistatic interactions. All these measures are related both to evolvability - deep chains show that fitnessincreasing paths are open-and to constraints-chains represent obligatory paths in evolution. This suggests an evolutionary interpretation of these peaks in terms of the tradeoff between evolvability (higher at low epistasis) and constraints (stronger with high epistasis). Previous measures that were shown numerically and by some analytical approximations to be non-monotonic include the total number of accessible paths (Szendro et al. 2013) and the number of exceedances, i.e. the number of available fitnessincreasing mutations after an evolutionary step (Neidhart et al. 2014), which are also related to evolvability and constraints. It is worth mentioning that chains and exceedance are both related to the out-degree distribution after one step of increasing fitness. This suggests that beyond the out-degree distribution, it is perhaps worth characterizing the sequences of out-degrees, e.g. the out-degree distribution along evolutionary paths.

Interestingly, many real landscapes tend to have longer chains than expected according to theoretical landscape models with random epistasis. This is the case for both experimental landscapes from Weinreich et al. (2006) and de Visser et al. (2009). This is especially apparent and interesting in the former landscape, since it implies some highly non-random structure of epistatic interactions for this set of mutations. This result has been found independently by randomization tests (Weinreich et al. 2013) and cannot be seen in complex measures of epistasis like the Fourier spectrum (Stadler 1996; Szendro et al. 2013) or the decay of correlations $\gamma_{\mathrm{d}}$ (Ferretti et al. 2016), however our chain measures were able to capture this signal. A possible explanation for this is the heterogeneity between the functional and selective effect of different mutations in real landscapes.

Chains are natural measures both from the evolutionary point of view and from the mathematical point of view. For most small empirical landscapes currently available (biallelic and with $L \approx 4-6$ loci), peaks and chains summarize most of the information about local evolutionary constraints and especially about the distribution of beneficial mutations. However, for larger landscapes or multi-allelic ones, other measures could be more informative about local and global constraints. This issue is discussed in details in the first two sections of the Appendix: in the first one we explain the reasons for the weak dependence of the number of chain steps on epistasis in terms of the behavior of the distribution of the number of beneficial mutations, while in the second we employ this framework to provide a guide to the choice of measures weakly related to epistasis in large multi-allelic landscapes. Global constraints can be also summarized by generalized chains, which are sets of "obligate" genotypes along evolutionary paths. These generalized chains describe non-local constraints and represent an interesting and potentially new source of information on the evolutionary dynamics in larger landscapes.

We are still far from predicting evolution on real landscapes based on their measures, partly because of the incomplete knowledge of the structure of real landscapes, and partly because of the lack of measures with a natural evolutionary interpretation. In the future, we expect to witness a strong increase in the number of publications of 

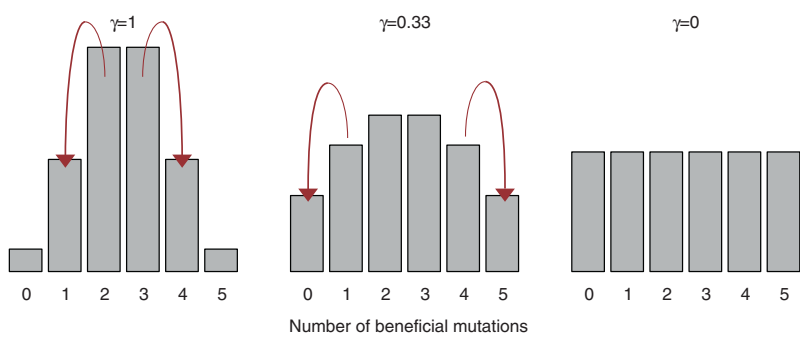

Fig. 6 Distribution of the number of beneficial mutations from random genotypes. Illustrated for biallelic RMF landscapes of size $L=5$ with increasing levels of epistasis from left (additive landscape) to right (House of Cards)

new experimentally resolved empirical landscapes. The measures that we propose here will therefore find applications in the understanding and classification of these landscapes, as well as in studies of model landscapes. The new chain measures are able to reveal information that is uncorrelated with the strength or amount of epistasis in the landscape, hence they will represent useful tools to discriminate and classify fitness landscapes. Chains also highlight the interplay of constraints and evolvability that influence evolution on complex landscapes.

Acknowledgements We thank Joachim Krug, Ivan Szendro, Johannes Neidhart, Arjan de Visser, Nick Barton, R. A. Watson for useful comments and discussions. The work was funded by grant ANR-12JSV7-0007 from Agence Nationale de la Recherche. LF is supported by funding from BBSRC grant BBS/E/I/00007039.

\section{Compliance with ethical standards}

Conflict of interest The authors declare that they have no conflict of interest.

\section{Appendix}

\section{Why are chains measures non-monotonic in epistasis?}

One of the most important features of chains is the low correlation, or non-monotonicity, of the number of chain steps versus epistasis. There is an intuitive explanation for this interesting property.

For many biological landscapes, as well as for classical models (like NK or RMF) the distribution of the number of beneficial mutations across genotypes (or equivalently, the out-degree distribution of the fitness graph) is approximately bell-shaped, with a small number of maxima and minima ( 0 and $L$ beneficial mutations respectively) and a large number of genotypes having as many beneficial as deleterious mutations.
The behavior of the distribution of beneficial mutations for the RMF at low, intermediate, and high epistasis is shown in Fig. 6 (the corresponding analytical formulae can be found in the next sections). It is easy to notice that the width of this distribution increases with the amount of epistasis. It will be shown elsewhere that in fact, the variance of this distribution is always proportional to the fraction of reciprocal sign epistasis in the landscape [Ferretti et al., in preparation]. For completely random landscapes (House of Cards), the distribution becomes flat on average.

Let us focus on genotypes that have few beneficial mutations (but not zero). Starting with an additive landscape and increasing the amount of epistasis, genotypes with few beneficial mutations are rare but increase steadily in number. At low epistasis, the increase is due to the flux of genotypes with intermediate numbers of beneficial mutations, which represent the most abundant class of genotypes. However, at high epistasis, genotypes with few beneficial mutations are more abundant and they tend to switch to peaks with increasing epistasis, hence they could decrease in number (see Fig. 6). Therefore, depending on the parameters, the number of genotypes with few beneficial mutations tends to show a non-monotonic behavior with respect to epistasis.

We are interested in small landscapes like the ones that have been experimentally resolved at present (biallelic, $L \approx$ 4-6). For these landscapes, the class of genotypes with few beneficial mutations is represented by the genotypes with $a$ single beneficial mutation, i.e. chain steps. The total number of chain steps in the landscape (i.e. the number of genotypes with a single beneficial mutation) is precisely the second component of the out-degree distribution, while the first component is the number of maxima. It is therefore a natural quantity of interest both mathematically and for its interpretation in terms of constraints and predictability of evolution.

\section{Generalizations for large, multi-allelic landscapes}

It follows from the arguments of the previous section that for larger landscapes or with more than two alleles per locus, the number of chains tends to grow rapidly with epistasis and the non-monotonicity is lost. The same reasoning suggests that a natural generalization of the number of chain steps is given by the components of the distribution of beneficial mutations that are non-monotonic in epistasis. The prediction of the biallelic RMF model for these components is shown in Fig. 7a. For not too large biallelic landscapes, the most non-monotonic component tends to have around $b \approx L / 3$ beneficial mutations. However, this could be model dependent and it is difficult to generalize to the multi-allelic case. Hence, we present a slightly different approach to this issue. 
A

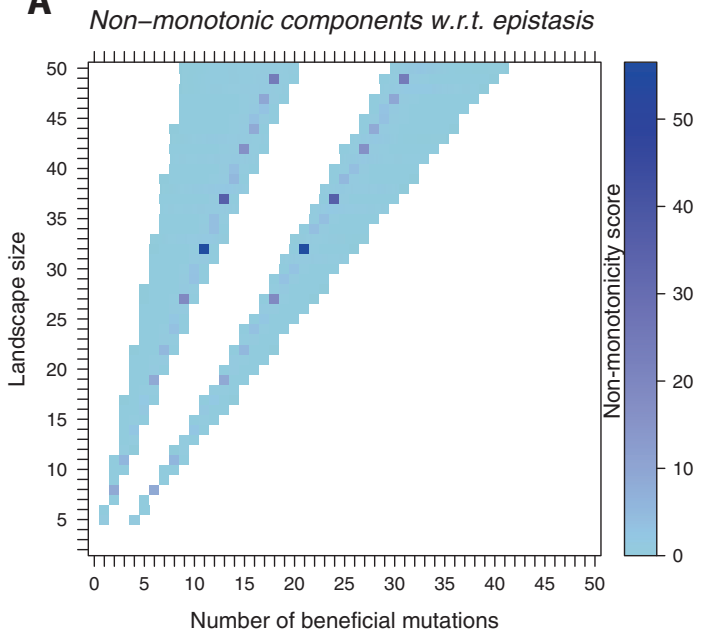

Fig. 7 Components of the distribution of beneficial mutations that are weakly dependent on epistasis. a Non-monotonicity (with respect to epistasis) of the components of the distribution of beneficial mutations, estimated for Rough Mount Fuji biallelic landscapes of different size with Gaussian House of Cards component. For a given number of loci in the landscape (vertical axis), only the colored components (horizontal axis) show some non-monotonicity. For example, for $L=5$, only the number of genotypes with 1 beneficial mutation (i.e. the number of chain steps) and with 4 beneficial mutations have a non-

In large multi-allelic landscapes, the size of almost all components of the distribution of beneficial mutations tends to differ between no epistasis and strong epistasis by a combinatorially large (or small) factor. In fact, in the biallelic case, this factor for the $b$ th component is $\frac{2^{L}}{(L+1)\left(\begin{array}{l}L \\ b\end{array}\right)}$. When this factor is very large or very small, the corresponding component is constrained to have an exponentially large dependence on epistasis. Hence, the only components that are not strongly correlated with epistasis are the ones that show minimal differences between no epistasis and strong epistasis. These "flattest" components are shown in Fig. $7 \mathrm{~b}$ as a function of the size of the landscape. We find again that chains (i.e. the component with a single beneficial mutation) correspond often to the flattest component for small landscapes. More precisely, they tend to be weakly dependent on epistasis for small biallelic landscapes of size $L=3-6$, as well as tri-allelic landscapes of size $L=3$ and tetra-allelic landscapes of size $L=2$.

It is possible to find an approximation for the flattest component in large landscapes. For a general additive landscape, the outdegree distribution is the one of the sum of $L$ integers between 0 and $A-1$ (which can be approximated by a normal distribution of mean $L(A-1) / 2$ and variance $\left.L\left(A^{2}-1\right) / 12\right)$, while for a strongly epistatic $\mathrm{HoC}$ landscape, the distribution is flat with all components of size $\frac{1}{L(A-1)+1}$. Hence, for $L(A-1)$ not too small, the "flattest"

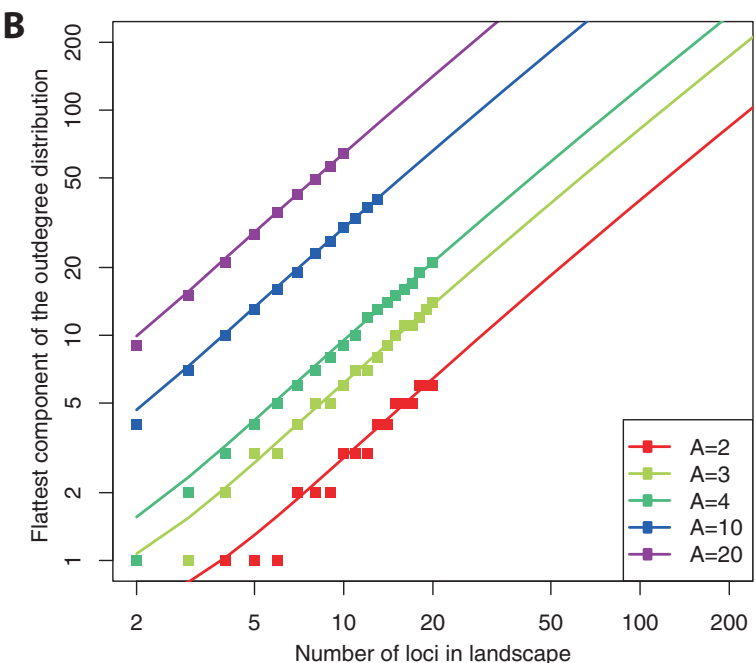

monotonic behavior. The amount of non-monotonicity $\frac{\max _{\gamma \in[0,1]} c(\gamma)-\min _{\gamma \in[0,1]} c(\gamma)}{|c(0)-c(1)|}-1$ is defined by the peak values of the component $c$ versus its values for no epistasis or for maximum epistasis. b "Flattest" component (defined as the one with minimal relative difference $\frac{|c(0)-c(1)|}{c(0)+c(1)}$ between no epistasis and maximum epistasis) as a function of the number of loci and the alleles per locus $A$ in the landscape. Intuitively, this component should be the least dependent on epistasis. (Here we report only the component with less beneficial mutations.) The lines show the approximation discussed in the text

components that depend weakly on epistasis will be concentrated around

$$
b \approx \frac{L(A-1)}{2}-\sqrt{\frac{L\left(A^{2}-1\right)}{12} \log \left(\frac{6 L(A-1)}{\pi(A+1)}\right)}
$$

beneficial mutations. The above formula can be used to find a statistical generalization of the number of chain steps for landscapes of arbitrary size.

Note that by symmetry, if a component $b<L(A-1) / 2$ is non-monotonic, genotypes with $L(A-1)-b$ beneficial mutations have similar properties of non-monotonicity with respect to epistasis, but they are less interesting from an evolutionary perspective, since they do not correspond to evolutionary constraints.

\section{Number of chain steps in the HoC model}

Given the linearity of expectations, the expected number of chain steps is given by the number of mutations in the landscape, $L \cdot 2^{L}$, multiplied by the probability that the mutation is a chain step. This is the probability that among the initial genotype and all its neighbors, the final one is the most fit and and the initial one is second in the fitness rank. Since fitness values are random and uncorrelated, the probability that a value is maximum among $L+1$ is $1 /$ $(L+1)$ and the conditional probability that another value is second is $1 / L$, therefore, we have that the average number of steps is $L \cdot 2^{L} / L(L+1)=2^{L} /(L+1)$. 


\section{Analytical results for chains in RMF}

We consider a RMF model with equal additive fitness contribution $s$ for each mutation, in addition to an $\mathrm{HoC}$ model with distribution $p(f)$ and cumulative distribution $C(f)=\int_{-\infty}^{f} p(x) \mathrm{d} x$.

Sorting the genotypes in order of their additive fitness, there are $\left(\begin{array}{l}L \\ k\end{array}\right)$ genotypes at the $k$ th level with $L-k$ mutations with positive additive fitness effect and $k$ with negative one. The average number of chain steps can be obtained as the sum over all possible steps of the probability of being a chain step. This probability depends on the distance $k$ of the genotype from the peak of the additive contribution. Given the HoC contribution $x$ to the fitness of the genotype, the probability can be expressed in terms of the probabilities that a mutation is beneficial $(1-C(x \pm s))$ and the others are deleterious $(C(x \pm s)$ each), considering all possible combinations of additive fitness effects. This should be integrated over the HoC contribution $x$ for the given genotype, then summed over all $\left(\begin{array}{l}L \\ k\end{array}\right)$ genotypes at distance $k$ and over all distances from 0 to $L$, obtaining

$$
\begin{aligned}
\text { \#steps }= & \sum_{k=0}^{L}\left(\begin{array}{l}
L \\
k
\end{array}\right)\left[k \int \mathrm{d} x p(x)(1-C(x+s)) C(x+s)^{k-1} C(x-s)^{L-k}\right. \\
& \left.+(L-k) \int \mathrm{d} x p(x)(1-C(x-s)) C(x-s)^{L-k-1} C(x+s)^{k}\right] \\
= & L \int d x p(x)[2-C(x-s)-C(x+s)] \cdot[C(x+s)+C(x-s)]^{L-1}
\end{aligned}
$$

The number of origins and of chain trees can be obtained in a similar way using the Cayley tree/Bethe lattice approximation. In this framework, we approximate locally the hypercube by a Cayley tree, i.e. a tree with $L$ branches at each node. This means that we neglect the overlapping between the next-to-nearest neighbors of the genotype considered and we assume them to be $(L-1)^{2}$ independent genotypes instead of $L(L-1) / 2$.

The probability that a genotype is the origin of a chain is product of the probability of having out-degree 1 and that all the other neighbors of lower fitness have out-degree different than 1 :

$$
\begin{aligned}
\text { \#origins }= & \sum_{k=0}^{L}\left(\begin{array}{l}
L \\
k
\end{array}\right)\left\{k \int \mathrm{d} x p(x)[1-C(x+s)][C(x+s)\right. \\
& \left.-p_{-}(x, s, k)\right]^{L-k}\left[C(x-s)-p_{+}(x, s, k)\right]^{k-1} \\
& +(L-k) \int \mathrm{d} x p(x)[1-C(x-s)][C(x-s) \\
& \left.\left.-p_{+}(x, s, k)\right]^{L-k-1}\left[C(x+s)-p_{-}(x, s, k)\right]^{k}\right\}
\end{aligned}
$$

where we define the probabilities that a neighbor genotype is the starting or ending point of a chain step ending at level $k$ :

$$
\begin{aligned}
& p_{+}(x, s, k)=\int \mathrm{d} y p(y) C(y+s)^{k} C(y-s)^{L-k-1} \\
& p_{-}(x, s, k)=\int \mathrm{d} y p(y) C(y+s)^{k-1} C(y-s)^{L-k}
\end{aligned}
$$

The probability that a genotype is the endpoint of a chain is the difference between the probability of being the final genotype of a chain step and the probability of being an intermediate point in a chain. The results are

$$
\begin{gathered}
\text { \#endpoints }=\sum_{k=0}^{L}\left(\begin{array}{l}
L \\
k
\end{array}\right)\left[1-\int \mathrm{d} x p(x)\left(1-p_{+}(x, s, k)\right)^{L-k} \cdot\left(1-p_{-}(x, s, k)\right)^{k}\right] \\
\left.\begin{array}{rl}
\text { \#intermediates } \\
\uparrow
\end{array}\right)=\sum_{k=0}^{L}\left(\begin{array}{l}
L \\
k
\end{array}\right)(L-k) \int \mathrm{d} x p(x)(1-C(x-s)) . \\
\cdot\left[C(x-s)^{L-k-1} C(x+s)^{k}\right. \\
-\left(C(x-s)-p_{+}(x, s, k)\right)^{L-k-1} \\
\\
\left.\cdot\left(C(x+s)-p_{-}(x, s, k)\right)^{k}\right]
\end{gathered}
$$

$\#$ intermediates $=\#$ intermediates $\uparrow_{\uparrow}(s)-\#$ intermediates $_{\uparrow}(-s)$

$\#$ chain trees $=\#$ endpoints $-\#$ intermediates

These formulae could be slightly simplified for distributions, where $C(f+s)$ can be expressed as a simple function of $C(f)$ : for example the exponential distribution $p(f)=e^{-f}$ with support $f>0$, which has $C(f)=1-e^{-f}$ and $C(f+s)=$ $1-e^{-s}(1-C(f))$, or the Gumbel distribution $p(f)=e^{-\left(f+e^{-f}\right)}$, in which case $C(f)=e^{-e^{-f}}$ and $C(f+$ $s)=C(f)^{e^{-s}}$ (Neidhart et al. 2014).

\section{Distribution of beneficial mutations in RMF models}

The full distribution of the number of beneficial mutations across genotypes in the RMF model can be obtained similarly to the number of chain steps, but with an additional summation over all possible ways to distribute $n$ beneficial mutations across $k$ mutations with a positive additive effect and $L-k$ mutations with a negative additive effect at level $k$, then dividing by the number $2^{-L}$ of genotypes:

$$
\begin{aligned}
p_{\text {ben }}(n)= & \frac{1}{2^{L}} \sum_{k=0}^{L}\left(\begin{array}{l}
L \\
k
\end{array}\right) \sum_{j=0}^{n} \int \mathrm{d} x p(x)\left(\begin{array}{c}
k \\
j
\end{array}\right)(1-C(x+s))^{j}(1-C(x-s))^{n-j} \\
& \cdot\left(\begin{array}{c}
L-k \\
n-j
\end{array}\right) C(x+s)^{k-j} C(x-s)^{L-k-n+j} \\
= & \frac{1}{2^{L}}\left(\begin{array}{c}
L \\
n
\end{array}\right) \int \mathrm{d} x p(x) \sum_{j=0}^{n}\left(\begin{array}{c}
n \\
j
\end{array}\right)(1-C(x+s))^{j}(1-C(x-s))^{n-j} \\
& \cdot \sum_{k-j=0}^{L-n}\left(\begin{array}{c}
L-n \\
k-j
\end{array}\right) C(x+s)^{k-j} C(x-s)^{L-k-n+j}
\end{aligned}
$$


and summing the binomials we obtain the simple result

$p_{\text {ben }}(n)=\left(\begin{array}{l}L \\ n\end{array}\right) \int \mathrm{d} x p(x)\left(1-\frac{C(x+s)+C(x-s)}{2}\right)^{n}\left(\frac{C(x+s)+C(x-s)}{2}\right)^{L-n}$

We can also obtain the number of maxima $N_{\text {peaks }}=2^{L} p_{\text {ben }}$ (0) (Neidhart et al. 2014):

$N_{\text {peaks }}=\int \mathrm{d} x p(x)[C(x+s)+C(x-s)]^{L}$

\section{References}

Achaz G, Rodriguez-Verdugo A, Gaut BS, Tenaillon O (2014) The reproducibility of adaptation in the light of experimental evolution with whole genome sequencing. Adv Exp Med Biol 781:211-231

Aita T, Uchiyama H, Inaoka T, Nakajima M, Kokubo T, Husimi Y (2000) Analysis of a local fitness landscape with a model of the rough mt. fuji-type landscape: application to prolyl endopeptidase and thermolysin. Biopolymers 54:64-79

Bank C, Matuszewski S, Hietpas RT, Jensen JD (2016) On the (un) predictability of a large intragenic fitness landscape. Proc Natl Acad Sci 113:14085-14090

Berestycki J,Brunet É,Shi Z, et al(2016) The number of accessible paths in the hypercube Bernoulli 22:653-680

Bridgham JT, Ortlund EA, Thornton JW (2009) An epistatic ratchet constrains the direction of glucocorticoid receptor evolution. Nature 461:515-519

Brouillet S, Annony H, Ferretti L, Achaz G (2015) Magellan: a tool to visualize small fitness landscapes. bioRxiv. Accessed date 31 Nov. 2017

Chevin LM, Martin G, Lenormand T (2010) Fisher's model and the genomics of adaptation: restricted pleiotropy, heterogenous mutation, and parallel evolution. Evolution 64:3213-3231

Chou HH, Chiu HC, Delaney NF, Segrè D, Marx CJ (2011) Diminishing returns epistasis among beneficial mutations decelerates adaptation. Science 332:1190-1192

Colegrave N, Buckling A (2005) Microbial experiments on adaptive landscapes. Bioessays 27:1167-1173

Crona K (2014) Polytopes, graphs and fitness landscapes. eds. H. Richter, A. Engelbrecht In: Recent advances in the theory and application of fitness landscapes. Springer, New York, pp. 177-205.

da Silva J, Coetzer M, Nedellec R, Pastore C, Mosier DE (2010) Fitness epistasis and constraints on adaptation in a human immunodeficiency virus type 1 protein region. Genetics 185:293-303

de Visser JA, Hoekstra RF, van den Ende H (1997) Test of interaction between genetic markers that affect fitness in aspergillus niger. Evolution 51:1499-1505

de Visser JAGM, Krug J (2014) Empirical fitness landscapes and the predictability of evolution. Nat Rev Genet 15:480-490

de Visser JAGM, Park SC, Krug J (2009) Exploring the effect of sex on empirical fitness landscapes Am Nat 174(Suppl 1):S15-S30

Ferretti L, Schmiegelt B, Weinreich D, Yamauchi A, Kobayashi Y, Tajima F et al. (2016) Measuring epistasis in fitness landscapes: the correlation of fitness effects of mutations. J Theor Biol 396:132-143
Flynn KM, Cooper TF, Moore FB, Cooper VS (2013) The environment affects epistatic interactions to alter the topology of an empirical fitness landscape. PLoS Genet 9:e1003426

Franke J, Klözer A, de Visser JAGM, Krug J (2011) Evolutionary accessibility of mutational pathways. PLoS Comput Biol 7: e1002134

Franke J, Krug J (2012) Evolutionary accessibility in tunably rugged fitness landscapes. J Stat Phys 148:706-723

Gillespie JH (1983) A simple stochastic gene substitution model. Theor Popul Biol 23:202-215

Hall DW, Agan M, Pope SC (2010) Fitness epistasis among 6 biosynthetic loci in the budding yeast saccharomyces cerevisiae. $\mathrm{J}$ Hered 101:S75-S84

Hegarty P, Martinsson A et al. (2014) On the existence of accessible paths in various models of fitness landscapes. Ann Appl Probab 24:1375-1395

Hwang S, Schmiegelt B, Ferretti L, Krug J (2017) Universality classes of interaction structures for nk fitness landscapes. Preprint at: arXiv: 170806556.

Jiang PP, Corbett-Detig RB, Hartl DL, Lozovsky ER (2013) Accessible mutational trajectories for the evolution of pyrimethamine resistance in the malaria parasite plasmodium vivax. J Mol Evol 77:81-91

Kauffman S (1993) The origins of order: self-organization and selection in evolution. Oxford University Press, New York

Kauffman SA, Weinberger ED (1989) The nk model of rugged fitness landscapes and its application to maturation of the immune response. J Theor Biol 141:211-245

Khan AI, Dinh DM, Schneider D, Lenski RE, Cooper TF (2011) Negative epistasis between beneficial mutations in an evolving bacterial population. Science 332:1193-1196

Kingman J,(1978) A simple model for the balance between selection and mutation J Appl Probab 15(01):1-12. https://doi.org/10.2307/ 3213231

Lobkovsky AE, Wolf YI, Koonin EV (2011) Predictability of evolutionary trajectories in fitness landscapes. PLoS Comput Biol 7: e1002302

Lozovsky ER, Chookajorn T, Brown KM, Imwong M, Shaw PJ, Kamchonwongpaisan S, et al. (2009) Stepwise acquisition of pyrimethamine resistance in the malaria parasite. Proc Natl Acad Sci 106:12025-12030

Lunzer M, Miller SP, Felsheim R, Dean AM (2005) The biochemical architecture of an ancient adaptive landscape. Science 310:499-501

Malcolm BA, Wilson KP, Matthews BW, Kirsch JF, Wilson AC (1990) Ancestral lysozymes reconstructed, neutrality tested and thermostability linked to hydrocarbon packing. Nature 345:86-89

Manhart M, Morozov AV (2014) Statistical physics of evolutionary trajectories on fitness landscapes. eds. R.Metzler, S.Redner, G. Oshanin In: First-passage phenomena and their applications. World Scientific, Singapore pp. 416-446

McCandlish DM (2011) Visualizing fitness landscapes. Evolution 65:1544-1558

Meini MR, Tomatis PE, Weinreich DM, Vila AJ (2015) Quantitative description of a protein fitness landscape based on molecular features. Mol Biol Evol 32:1774-1787

Mira PM, Meza JC, Nandipati A, Barlow M (2015) Adaptive landscapes of resistance genes change as antibiotic concentrations change. Mol Biol Evol 32:2707-2715

Neidhart J, Szendro IG, Krug J (2014) Adaptation in tunably rugged fitness landscapes: the Rough Mount Fuji model. Genetics 198:699-721

O'maille PE, Malone A, Dellas N, Hess BA, Smentek L, Sheehan I, et al. (2008) Quantitative exploration of the catalytic landscape separating divergent plant sesquiterpene synthases. Nat Chem Biol 4:617-623 
Orr HA (2005) The genetic theory of adaptation: a brief history. Nat Rev Genet 6:119-127

Palmer AC, Toprak E, Baym M, Kim S, Veres A, Bershtein S, et al. (2015) Delayed commitment to evolutionary fate in antibiotic resistance fitness landscapes. Nat Commun 6:7385

Salverda MLM, Dellus E, Gorter FA, Debets AJM, van der Oost J, Hoekstra RF, et al. (2011) Initial mutations direct alternative pathways of protein evolution. PLoS Genet 7:e1001321

Schmiegelt B, Krug J (2014) Evolutionary accessibility of modular fitness landscapes. J Stat Phys 154:334-355

Stadler PF (1996) Landscapes and their correlation functions. J Math Chem 20:1-45

Stadler PF (2002) Fitness landscapes. eds. M.Lassig, A.Valleriani In: Biological evolution and statistical physics. Springer, pp 183-204

Szendro IG, Schenk MF, Franke J, Krug J, de Visser JAGM (2013) Quantitative analyses of empirical fitness landscapes. J Stat Mech. 1: P01005

Tan L, Serene S, Chao HX, Gore J (2011) Hidden randomness between fitness landscapes limits reverse evolution. Phys Rev Lett 106:198102
Weinberger ED (1991) Local properties of kauffman's n-k model: a tunably rugged energy landscape. Phys Rev A 44:6399

Weinreich DM, Delaney NF, Depristo MA, Hartl DL (2006) Darwinian evolution can follow only very few mutational paths to fitter proteins. Science 312:111-114

Weinreich DM, Lan Y, Jaffe J, Heckendorn RB (2017) The influence of higher-order epistasis on biological fitness landscape topography. bioRxiv. https://www.biorxiv.org/content/early/2017/07/ 18/164798 Accessed date 31 Nov. 2017.

Weinreich DM, Lan Y, Wylie CS, Heckendorn RB (2013) Should evolutionary geneticists worry about higher-order epistasis? Curr Opin Genet Dev 23:700-707

Weinreich DM, Watson RA, Chao L (2005) Perspective: sign epistasis and genetic constraint on evolutionary trajectories. Evolution 59:1165-1174

Whitlock MC, Bourguet D (2000) Factors affecting the genetic load in drosophila: synergistic epistasis and correlations among fitness components. Evolution 54:1654-1660

Wright S (1932) The roles of mutation, inbreeding, crossbreeding and selection in evolution. Proc 6th Int Cong Genet 1:356-366 